\title{
STRESS FIELD DISTRIBUTION IN MAGNETOELASTIC PRESSURE FORCE SENSOR
}

Modelling of the stress field distribution in a magnetoelastic sensor core is proposed. The components of stress field are carried out by a numerical solution of boundary-value problem for stress field. The obtained results are presented in graphical form.

\section{Introduction}

The magnetoelastic pressure force sensor operates on the Villari effect principle. The Villari effect, also called a stress-magnetization, is based on the change of permeability of a ferromagnetic body exposed to a mechanical force, which results in mechanical stress within the body. The permeability increment is proportional to that component of mechanical stress, which is collinear to magnetic field vectors [1].

The modelling of the magnetic field distribution in the core of such a sensor with respect to the variation of the permeability due to mechanical stress requires to determine the stress field distribution.

In the general case, the planar stress field is unambiguously determined by three components, two perpendicular components and a shear component [2]. These components can be appointed by various methods. In this paper a method based on a numerical solution of boundary-value problem is proposed. This task was solved by professional code PDE Toolbox in package MATLAB.

\section{Magnetoelastic pressure force sensor}

The arrangement of the magnetoelastic pressure force sensor is depicted in Fig. 1. The sensor core consists of fifty ferromagnetic transformer sheets, thickness of each being $0.5 \mathrm{~mm}$. Each sheet contains four holes of the radius of $1 \mathrm{~mm}$. The conductors of primary (10 turns $/ 0.35 \mathrm{~mm}$ ) and secondary windings ( 8 turns $/ 0.25 \mathrm{~mm}$ ) are led through these holes collaterally. The sensor is assigned for the measuring of nominal pressure force $120 \mathrm{kN}$ that is equivalent to $100 \mathrm{MPa}$. A simplifying representation of the sensor core is in Fig. 2.

If Cartesian coordinate system is applied to such a simplified form of sensor core (see Fig. 2), the current density vector has

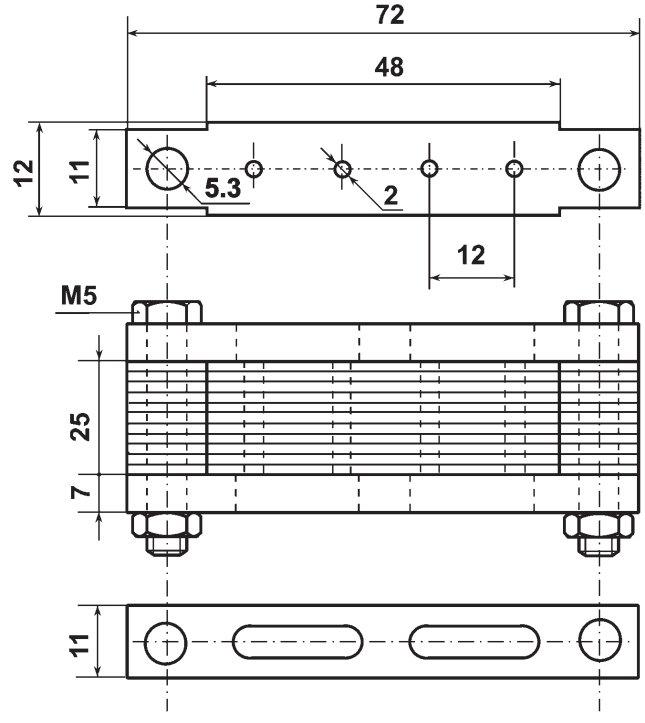

Fig. 1 The magnetoelastic pressure force sensor

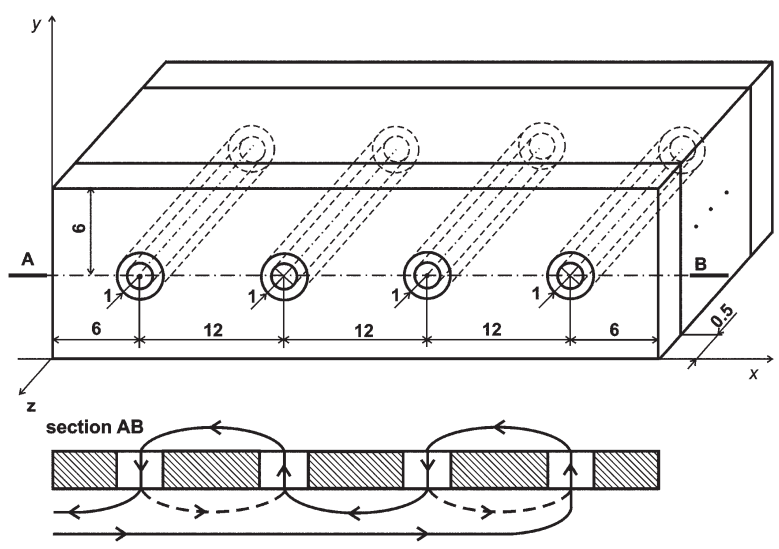

Fig. 2. The magnetoelastic pressure force sensor core in simplified form

\footnotetext{
* Iveta Tomcikova ${ }^{1}$, Dobroslav Kovac ${ }^{1}$, Irena Kovacova ${ }^{2}$

${ }^{1}$ Department of Theoretical Electrotechnics and Electrical Measurement, Faculty of Electrical Engineering and Informatics, Technical University of Kosice, Slovakia, E-mail: iveta.tomcikova@tuke.sk

${ }^{2}$ Department of Electrical Engineering, Mechatronics and Industrial Engineering, Faculty of Electrical Engineering and Informatics, Technical University of Kosice, Slovakia
} 
a nonzero component only in the z-direction. This component produces magnetic field whose vectors have nonzero components only in the directions $x$ and $y$ [3].

Moreover, the dimensions of the sheet are designed in such a way that the sheet can be divided into four squared shapes, each with a circle hole in the middle. A conductor (the cross-section of which is the same as the cross-section of the primary windings conductors) replaces the primary winding. One of such shapes is called the integrative sensor element (Fig. 3left). With respect to the symmetry of the sheet it is sufficient to determine the magnetic field distribution in the integrative sensor element only. The stress field distribution is necessary to determine only in a part of the integrative sensor element that is made of a ferromagnetic material (see Fig. 3right).

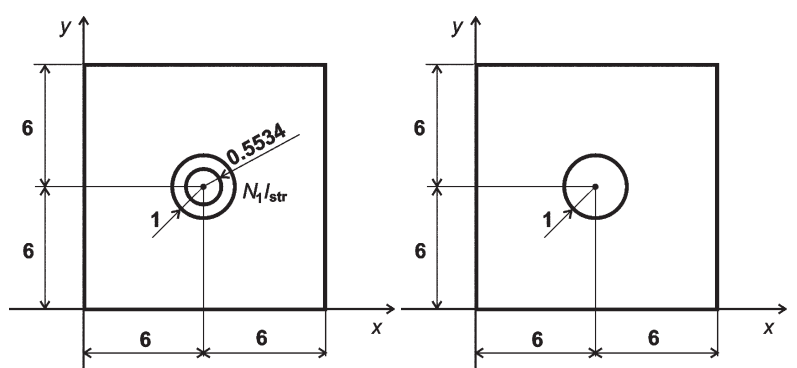

Fig. 3 The integrative sensor element; left: for determination the magnetic field distribution, right: for determination the stress field distribution

For the modelling of the magnetic field distribution in the sensor core with respect to the variation of material permeability due to mechanical stress it is necessary to investigate only the $x$ direction stress $\left(\sigma_{x}\right)$ and y-direction stress $\left(\sigma_{y}\right)$ in the integrative sensor element (see Fig. 4).

\section{PDE system formulation for plane stress}

The determination of stress field in the integrative sensor element can be formulated as a boundary-value problem in terms of the displacement components. The first of all it is essential to formulate the PDE system for balance of force in terms of the displacement components with the boundary conditions.

For plane stress, in the body made of the homogeneous isotropic material, the balance of force equations are [2]:

$$
\begin{aligned}
& \frac{\partial \sigma_{x}}{\partial x}+\frac{\partial \tau_{x y}}{\partial y}+X=0, \\
& \frac{\partial \sigma_{y}}{\partial y}+\frac{\partial \tau_{x y}}{\partial x}+Y=0,
\end{aligned}
$$

where $\sigma_{x}$ is the $x$-direction stress, $\sigma_{y}$ being the y-direction stress, $\tau_{x y}$ being the shear stress and $X, Y$ being the volume forces.

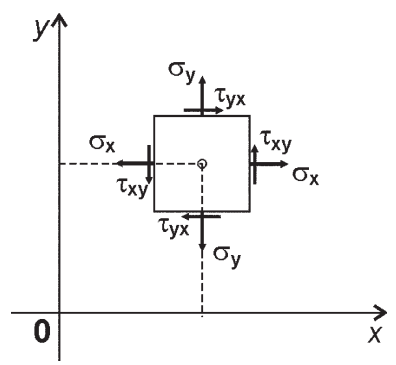

Fig. 4 The stress components in the xy plane

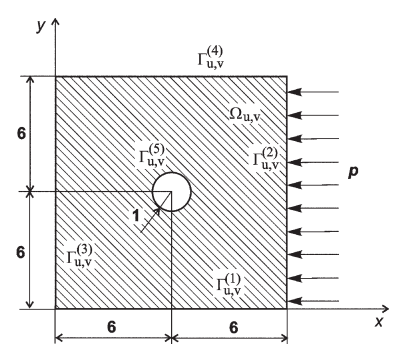

Fig. 5 The domain $\Omega_{u, v}$
The stress components are closely related to the strain components and these relations are defined by Hooke law

$$
\left[\begin{array}{l}
\sigma_{x} \\
\sigma_{y} \\
\tau_{x y}
\end{array}\right]=\frac{E}{1-\nu^{2}}\left[\begin{array}{ccc}
1 & \nu & 0 \\
\nu & 1 & 0 \\
0 & 0 & \frac{1-\nu}{2}
\end{array}\right]\left[\begin{array}{l}
\varepsilon_{x} \\
\varepsilon_{y} \\
\gamma_{x y}
\end{array}\right]
$$

where $E$ is Young's modulus, $v$ being Poisson's ratio,

The strain components can be expressed by the displacements, $u$ the displacement in the $x$-direction and $v$ the displacement in the $y$-direction

$$
\varepsilon_{x}=\frac{\partial u}{\partial x}, \varepsilon_{y}=\frac{\partial v}{\partial y}, \gamma_{x y}=\frac{\partial u}{\partial y}+\frac{\partial v}{\partial x},
$$

where $\varepsilon_{x}$ is the $x$ - direction strain, $\varepsilon_{y}$ being the $y$ - direction strain, $\gamma_{x y}$ being the shear strain.

Combining the equations (1) - (4) and assuming that there are no volume forces, we arrived at the PDE system for balance of force in terms of the displacement components. The system takes form

$$
\begin{aligned}
& \frac{E}{1-\nu^{2}} \frac{\partial^{2} u}{\partial x^{2}}+\frac{E}{2(1+\nu)} \frac{\partial^{2} u}{\partial y^{2}}+\nu \frac{E}{1-\nu^{2}} \frac{\partial^{2} v}{\partial x \partial y}+ \\
& +\frac{E}{2(1+\nu)} \frac{\partial^{2} v}{\partial y \partial x}=0, \\
& \frac{E}{2(1+\nu)} \frac{\partial^{2} v}{\partial x^{2}}+\frac{E}{1-\nu^{2}} \frac{\partial^{2} v}{\partial y^{2}}+\frac{E}{2(1+\nu)} \frac{\partial^{2} u}{\partial x \partial y}+ \\
& +\nu \frac{E}{1-\nu^{2}} \frac{\partial^{2} u}{\partial y \partial x}=0,
\end{aligned}
$$

From mathematical point of view, the equations (5) and (6) represent a system of two PDE of elliptic type.

The following boundary conditions for the system PDE can be given:

- For each point on the boundary is specified the value of the displacement

$$
u=g_{1}, v=g_{2} .
$$

This boundary condition is called Dirichlet boundary condition. 
- For each point on the boundary is known the surface load (the derivative of the displacement by the outward normal vector)

$$
\begin{gathered}
\boldsymbol{e}_{n}\left(\left[\begin{array}{cc}
\frac{E}{1-\nu^{2}} & 0 \\
0 & \frac{E}{2(1+\nu)}
\end{array}\right] \nabla u\right)+\boldsymbol{e}_{n}\left(\left[\begin{array}{cc}
0 & \frac{\nu E}{1-\nu^{2}} \\
\frac{E}{2(1+\nu)} & 0
\end{array}\right] \nabla v\right)=p_{x}, \\
\boldsymbol{e}_{n}\left(\left[\begin{array}{cc}
0 & \frac{E}{2(1+\nu)} \\
\frac{v E}{1-\nu^{2}} & 0
\end{array}\right] \nabla u\right)+\boldsymbol{e}_{n}\left(\left[\begin{array}{cc}
\frac{E}{2(1+\nu)} & 0 \\
0 & \frac{E}{1-\nu^{2}}
\end{array}\right] \nabla \nu\right)=p_{y},
\end{gathered}
$$

where $e_{n}$ is the outward normal vector of the boundary, $\nabla$ being Hamilton operator, $p_{x}$ being the pressure in the direction of $x$-axis, $p_{y}$ being the pressure in the direction of $y$-axis.

This boundary condition is called Neumann boundary condition.

\section{Boundary-value problem formulation}

The boundary-value problem formulation also requires determining the domain, in which the stress field will be solved. The integrative sensor element has the squared shape with a circle hole in the middle (see Fig. 4). It can be considered to be a planar body with the following properties: dimension 12-by-12 mm, thickness $0.5 \mathrm{~mm}$ and the radius of the circle hole $1 \mathrm{~mm}$. The element is made of ferromagnetic transformer sheet, whose parameters are: $E=1.86 .105 \mathrm{MN} / \mathrm{m}^{2}, v=0.3$. The element is exposed to a constant continuous pressure stress $\boldsymbol{p}=-p_{x} \boldsymbol{e}_{x}$ ( $\boldsymbol{e}_{x}$ being the unit vector in the direction of $\mathrm{x}$-axis).

The domain $\Omega_{u, v}$, in which the stress field will be solved, is surrounded by five boundaries $\Gamma_{u, v}^{(1)}, \Gamma_{u, v}^{(2)}, \Gamma_{u, v}^{(3)}, \Gamma_{u, v}^{(4)}$, a $\Gamma_{u, v}^{(5)}$ (see Fig. 5). The boundary conditions for the displacement components are following:

- The boundary $\Gamma_{u, v}^{(2)}$ is subjected to a pressure $\boldsymbol{p}=-p_{x} \boldsymbol{e}_{x}$ :

$$
\begin{aligned}
& \frac{E}{1-\nu^{2}} \frac{\partial u}{\partial x}+\frac{\nu E}{1-\nu^{2}} \frac{\partial v}{\partial y}=-p_{x}, \\
& \frac{\partial u}{\partial y}+\frac{\partial v}{\partial x}=0 .
\end{aligned}
$$

- There is no displacement on the boundary $\Gamma_{u, v}^{(3)}$ :

$$
u=0, \quad v=0,
$$

- The remaining boundaries $\Gamma_{u, v}^{(1)}, \Gamma_{u, v}^{(4)}$ a $\Gamma_{u, v}^{(5)}$ are free (no normal stress):

$$
\begin{aligned}
& \frac{\partial u}{\partial y}+\frac{\partial v}{\partial x}=0, \\
& \nu \frac{\partial u}{\partial x}+\frac{\partial v}{\partial y}=0 .
\end{aligned}
$$

The investigated boundary-value problem was solved by using a professional code PDE Toolbox. The toolbox is based on the finite element method (FEM). The solution was made by using the adaptive mode option, which enables to refine the mesh in areas where the gradient of the solution is large in order to increase the accuracy of the solution [7]. The obtained results (for generated triangular mesh consisting of 42112 nodes and 83456 triangles) at the pressure force $p_{x}=8,23 \mathrm{MPa}$ are depicted in Figs. $6-11$. In these figures are plotted the contour lines, e.g. the lines connected points of equal value of the depicted quantity in the domain $\Omega_{u, v}$. In Figs. 6 and 7 are visualised the displacements $u(x, y)$ in the $x-$ direction and $v(x, y)$ in the $y$ - direction. The $x$ - direction strain and the $y$-direction strain are depicted in Figs. 8 and 9. The $x-$ direction stress and the $y$ - direction stress are shown in Figs. 10 and 11 .

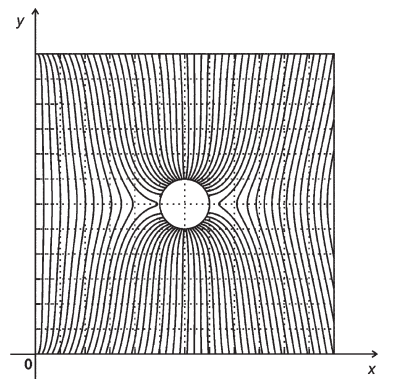

Fig. 6 The displacement $u$ in the $x$-direction

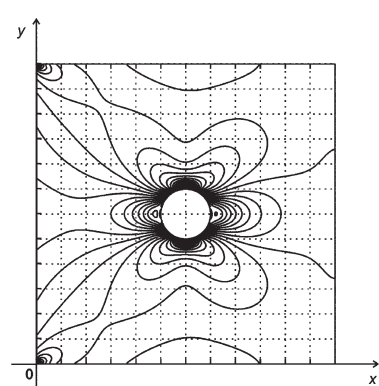

Fig. 8 The strain $\varepsilon_{x}$ in the $x$ - direction

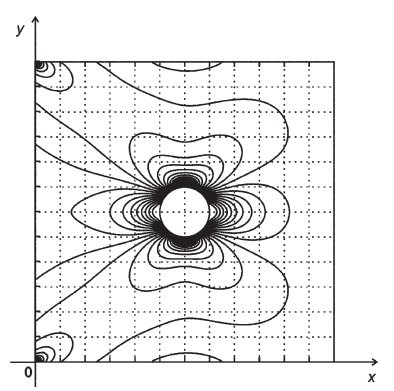

Fig. 10 The stress $\sigma_{x}$ in the $x$ - direction

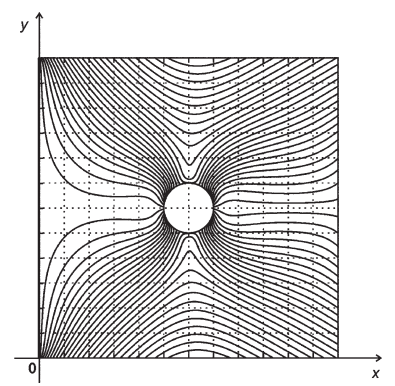

Fig. 7 The displacement $v$ in the $y$ - direction

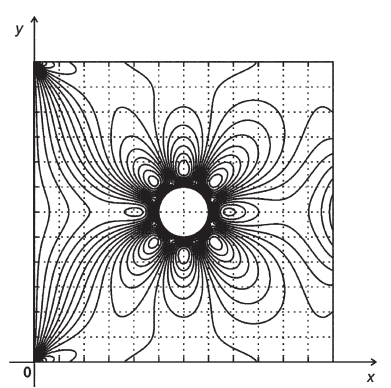

Fig. 9 The strain $\varepsilon_{y}$ in the $y$ - direction

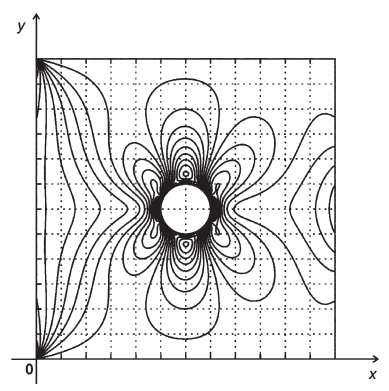

Fig. 11 The stress $\sigma_{y}$ in the $y$ - direction 


\section{Conclusion}

The paper is aimed at assignment of stress field distribution in the magnetoelastic pressure force sensor. The task was solved as the boundary-value problem in terms of the displacement components by the professional code PDE Toolbox in package MATLAB. The toolbox provides a powerful and flexible environment not only for the solution of elliptic, parabolic and hyperbolic partial differential equations, but it also handles the solution of a system of partial differential equations. The normal components of the stress field and also the components of the strain and displacement were visualised.

\section{Acknowledgements}

The paper was prepared under the support of Slovak grant projects VEGA No. 1/4174/07, VEGA No. 1/0660/08, KEGA No. 3/5227/07, KEGA No. 3/6386/08 and KEGA No. 3/6388/08.

\section{References}

[1] ZEHNULA, K.: Sensors of Non-electric Quantities, SNTL, Prague 1977.

[2] TREBUNA, F., JURICA, V., SIMCAK, F.: Elasticity and Strength I., Edition of scientific and special literature. Košice 2000.

[3] MAYER, D., ULRYCH, B.: Fundamentals of Electric and Magnetic Fields Numerical Calculations, SNTL, Prague 1988.

[4] TREBUNA, F., JURICA, V., SIMCAK, F.: Elasticity and Strength II.. Edition of Scientific and Special Literature, Košice 2000.

[5] MUSCHELISVILI, N. I.: Some Fundamental Problems of Mathematical Stress Theory, Publishing House of Science Academy USSR, Moscow 1954.

[6] SAVIN, G.: Stress Field Distribution around Holes, Nauka dumka, Kiev 1968.

[7] Partial Differential Equation Toolbox User's Guide. 